\title{
SOME REMARKS ABOUT ELEMENTARY DIVISOR RINGS $\left({ }^{1}\right)$
}

\author{
BY \\ LEONARD GILLMAN AND MELVIN HENRIKSEN
}

In this and the following paper [2], we are concerned with obtaining conditions on a commutative ring $S$ with identity element in order that every matrix over $S$ can be reduced to an equivalent diagonal matrix $\left({ }^{2}\right)$. Following Kaplansky [4], we call such rings elementary divisor rings. A necessary condition is that $S$ satisfy

F: all finitely generated ideals are principal.

It has been known for some time that if $S$ satisfies the ascending chain condition on ideals, and has no zero-divisors, then $\mathbf{F}$ is also sufficient. Helmer [3] showed that the chain condition can be replaced by the less restrictive hypothesis that $S$ be adequate (i.e., of any two elements, one has a "largest" divisor that is relatively prime to the other $\left.\left({ }^{2}\right)\right)$. Kaplansky [4] generalized this further by permitting zero-divisors, provided that they are all in the (Perlis-Jacobson) radical.

By a slight modification of Kaplansky's argument, we find that the condition on zero-divisors can be replaced by the hypothesis that $S$ be an Hermite ring (i.e., every matrix over $S$ can be reduced to triangular form $\left({ }^{2}\right)$ ). This is an improvement, since, in any case, it is necessary that $S$ be an Hermite ring, while, on the other hand, it is not necessary that all zerodivisors be in the radical. In fact, we show that every regular commutative ring with identity is adequate. However, the condition that $S$ be adequate is not necessary either.

We succeed in obtaining a necessary and sufficient condition that $S$ be an elementary divisor ring. Along the way, we obtain a necessary and sufficient condition that $S$ be an Hermite ring. In the paper that follows [2], we make constant use of these results. In particular, we construct examples of rings that satisfy $\mathbf{F}$ but are not Hermite rings, and examples of Hermite rings that are not elementary divisor rings. However, all these examples contain zero-divisors; therefore, the question as to whether there exist corresponding examples that are integral domains is left unsettled.

Definition 1 . An $m$ by $n$ matrix $A$ over $S$ admits triangular reduction if

Presented to the Society, December 28, 1954 under the title Concerning adequate rings and elementary divisor rings. II ; received by the editors April 20, 1955 and, in revised form, November 14, 1955.

(1) The preparation of this paper was sponsored (in part) by the National Science Foundation, under grant NSF G1129.

(2) The precise definition is given below. 
there exist nonsingular $\left(^{3}\right)$ matrices $U, V$ such that $A U=\left[b_{i, j}\right]$ is triangular (i.e., $b_{i, j}=0$ whenever $i<j$ ), and $V A$ is triangular; $A$ admits diagonal reduction if there exist nonsingular matrices $P, Q$ such that $P A Q=\left[c_{i, j}\right]$ is diagonal (i.e., $c_{i, j}=0$ whenever $i \neq j$ ), and every $c_{i, i}$ is a divisor of $c_{i+1, i+1}[4]\left({ }^{4}\right)$.

THEOREM 2 (KAPLANSKY). Let $S$ be a commutative ring with identity. If all 1 by 2 and all 2 by 1 matrices over $S$ admit diagonal reduction, then every matrix over $S$ admits triangular reduction; in this case, $S$ is called an Hermite ring. If, in addition, all 2 by 2 matrices over $S$ admit diagonal reduction, then every matrix over $S$ admits diagonal reduction; in this case, $S$ is called an elementary divisor ring.

For the proof, see [4, Theorems 3.5 and 5.1].

Obviously, every elementary divisor ring is an Hermite ring. Furthermore, every Hermite ring satisfies F [4, p. 465].

In order to prove that a given commutative ring is an Hermite ring, it suffices, by symmetry, to show only that every 1 by 2 matrix admits diagonal reduction.

ThEOREM 3. A commutative ring $S$ with identity is an Hermite ring if and only if it satisfies the condition

$\mathrm{T}:$ for all $a, b \in S$, there exist $a_{1}, b_{1}, d \in S$ such that $a=a_{1} d, b=b_{1} d$, and $\left(a_{1}, b_{1}\right)=(1)$.

Proof. Suppose that $S$ satisfies T. In order to show that $S$ is an Hermite ring, it suffices to show that every 1 by 2 matrix $[a b]$ admits diagonal reduction (Theorem $2 \mathrm{ff}$.). Let $a_{1}, b_{1}, d, s, t$ satisfy $a=a_{1} d, b=b_{1} d$, and $s a_{1}+t b_{1}=1$. Let

$$
Q=\left[\begin{array}{rr}
s & -b_{1} \\
t & a_{1}
\end{array}\right]
$$

Then $Q$ is nonsingular, and $[a b] Q=\left[\begin{array}{ll}a & 0\end{array}\right]$.

Conversely, suppose that $S$ is an Hermite ring. Let $a, b \in S$. By hypothesis, there exists a nonsingular matrix $Q$, which we denote as in (1), such that $\left[\begin{array}{ll}a & b\end{array}\right] Q=\left[\begin{array}{ll}d & 0\end{array}\right]$ for some $d \in S$. Then $a b_{1}=b a_{1}$, and $s a+t b=d$. Since $Q$ is nonsingular, we may assume that $s a_{1}+t b_{1}=1$. Then $s a_{1} a+t b_{1} a=a$, whence $s a_{1} a+t a_{1} b=a$, i.e., $a_{1} d=a$. Similarly, $b_{1} d=b$.

The following lemma, due essentially to Kaplansky $[4, \S 4]$, shows that in dealing with condition $\mathbf{T}$ relative to any specific pair $a, b$, it suffices to consider any particular generator of the ideal $(a, b)$.

Lemma 4. Let $a, b \in S$. If $a_{1}, b_{1}, d$ exist as in condition $\mathbf{T}$ (whence $(a, b)=(d)$ ),

${ }^{(3)}$ By nonsingular, we mean that $U$ (resp. $V$ ) has a two-sided inverse in the ring of all $n$ by $n$ (resp. $m$ by $m$ ) matrices over $S$.

(4) Kaplansky [4] does not require commutativity of $S$. 
then for all $d^{\prime}$ with $(a, b)=\left(d^{\prime}\right)$, there exist $a_{1}^{\prime}, b_{1}^{\prime}$ such that $a=a_{1}^{\prime} d^{\prime}, b=b_{1}^{\prime} d^{\prime}$, and $\left(a_{1}^{\prime}, b_{1}^{\prime}\right)=(1)$.

Proof. Write $d=k d^{\prime}, d^{\prime}=l d$, and choose $s, t$ such that $s a_{1}+t b_{1}=1$. Define $a_{1}^{\prime}=k l t-t+a_{1} k$, and $b_{1}^{\prime}=s-k l s+b_{1} k$. Then $a_{1}^{\prime} d^{\prime}=a, b_{1}^{\prime} d^{\prime}=b$, and $\left(s l-b_{1}\right) a_{1}^{\prime}$ $+\left(t l+a_{1}\right) b_{1}^{\prime}=1$.

As a straightforward consequence of Lemma 4, we have:

Corollary 5. If $S$ satisfies condition $\mathbf{T}$, then given $a, b, c, d$ with $(a, b, c)$ $=(d)$, there exist $a_{1}, b_{1}, c_{1}$ such that $a=a_{1} d, b=b_{1} d, c=c_{1} d$, and $\left(a_{1}, b_{1}, c_{1}\right)=(1)$.

Theorem 6. A commutative ring $S$ with identity is an elementary divisor ring if and only if it is an Hermite ring that satisfies the condition

$\mathbf{D}^{\prime}:$ for all $a, b, c \in S$ with $(a, b, c)=(1)$, there exist $p, q \in S$ such that ( $p a$, $p b+q c)=(1)$.

Thus, $\mathrm{S}$ is an elementary divisor ring if and only if it satisfies $\mathbf{T}$ and $\mathbf{D}^{\prime}$.

Proof. We have already remarked that every elementary divisor ring is an Hermite ring. The necessity of the condition $\mathbf{D}^{\prime}$ is established in the proof of $[4$, Theorem 5.2$]$.

The sufficiency of the two conditions is obtained by making the following two changes in the proof of [4, Theorem 5.2]. First, delete the reference to [4, Theorem 3.2]. Second, justify the fact that $x a_{1}+y b_{1}+z c_{1}$ is a unit by referring to our Corollary 5.

Definition 7 (Helmer) $\left.{ }^{5}\right)$. A commutative ring $S$ with identity is said to be adequate if it satisfies the two conditions $\mathrm{F}$ and

A: for every $a, b \in S$, with $a \neq 0$, there exist $a_{1}, d \in S$ such that (i) $a=a_{1} d$, (ii) $\left(a_{1}, b\right)=(1)$, and (iii) for every nonunit divisor $d^{\prime}$ of $d$, we have $\left(d^{\prime}, b\right) \neq(1)$. If in the proof of $[4$, Theorem 5.3$]$, we replace the reference to $[4$, Theorem 5.2] by a reference to our Theorem 6 , we obtain:

THEOREM 8. An adequate ring is an elementary divisor ring if and only if it is an Hermite ring.

Definition 9 (von Neumann) $\left.{ }^{6}\right)$. A commutative ring $S$ with identity is said to be regular if for every $a \in S$, there exists $x \in S$ such that $a^{2} x=a$.

von Neumann [5] shows that in any regular ring, every principal ideal is generated by an idempotent; in fact, if $a^{2} x=a$, then $e=a x$ is idempotent, and $(a)=(e)$. Furthermore, every finitely generated ideal is principal; for if $b^{2} y=b, f=b y$, and $d=e+f-e f$, then $a=a d, b=b d$, and $d \in(e, f)=(a, b)$,

(5) Helmer's definition [3] was restricted to integral domains. More general commutative rings with identity were first investigated in this connection by Kaplansky [4].

(6) In von Neumann's definition [5], it is not assumed that $S$ be commutative. The defining condition in the general case is $a x a=a$. 
whence $(a, b)=(d)$. Moreover, every element is a unit multiple of an idempotent $\left({ }^{7}\right)$ :

Lemma 10. For any element a of a regular ring $S$ (commutative, with identity), there exists $a$ unit $u$ such that $a^{2} u=a$ (whence $e=a u$ is idempotent).

Proof. Let $x$ satisfy $a^{2} x=a$, and let $z$ satisfy $x^{2} z=x$. Define $u=1+x-x z$. Since $a x z=\left(a^{2} x\right) x z=a^{2} x=a$, we have $a^{2} u=a$. Now obviously, $(u, x)=(1)$. But $x u=x^{2}$, whence $x$ belongs to every maximal ideal that contains $u$. It follows that $u$ is a unit.

THEOREM 11. Every regular ring $S$ (commutative, with identity) is adequate.

Proof. We have already remarked that $S$ must satisfy F. In order to show that $S$ satisfies A, consider any $a, b \in S$. By Lemma 10, we may work instead with the idempotents $e, f$ of which $a, b$ are unit multiples. Define $d=e+f-e f$; then, as noted above, $(d)=(e, f)$. Put $e_{1}=1-f+e f$. Then $e=e_{1} d$ and $\left(e_{1}, f\right)$ $=(1)$. Since $d$ divides $f$, no nonunit divisor $d^{\prime}$ of $d$ can be relatively prime to $f$.

REMARK 12. Kaplansky points out [4, p. 474] that by using results developed in [1], one can show that every commutative regular ring $S$ with identity is an elementary divisor ring. This can also be seen as follows. Working again with the idempotents $e$ and $f$, let $d$ and $e_{1}$ be as above, and define $f_{1}=f$. Then $e=e_{1} d, f=f_{1} d$, and $\left(e_{1}, f_{1}\right)=(1)$. It follows that $S$ is an Hermite ring (Theorem 3 ). Therefore, by Theorem $8, S$ is an elementary divisor ring.

\section{REFERENCES}

1. R. Arens and I. Kaplansky, Topological representations of algebras, Trans. Amer. Math. Soc. vol. 63 (1948) pp. 457-481.

2. L. Gillman and M. Henriksen, Rings of continuous functions in which every finitely generated ideal is principal, Trans. Amer. Math. Soc. vol. 82 (1956) pp. 368-393.

3 . $\mathrm{O}$. Helmer, The elementary divisor theorem for certain rings without chain condition, Bull. Amer. Math. Soc. vol. 49 (1943) pp. 225-236.

4. I. Kaplansky, Elementary divisors and modules, Trans. Amer. Math. Soc. vol. 66 (1949) pp. 464-491. 713.

5. J. von Neumann, On regular rings, Proc. Nat. Acad. Sci. U.S.A. vol. 22 (1936) pp. 707-

Purdue University,

LAFAYETTE, IND.

(7) The arguments that follow are motivated by [1, Theorem 2.2]. 